\title{
On Local Definitions of Length of Digital Curves
}

\author{
Mohamed Tajine and Alain Daurat \\ LSIIT UMR 7005 CNRS-ULP, Pôle API, Boulevard Sébastien Brant, \\ 67400 Illkirch-Graffenstaden, France \\ \{tajine, daurat\}@lsiit.u-strasbg.fr
}

\begin{abstract}
In this paper we investigate the 'local' definitions of length of digital curves in the digital space $r \mathbb{Z}^{2}$ where $r$ is the resolution of the discrete space. We prove that if $\mu_{r}$ is any local definition of the length of digital curves in $r \mathbb{Z}^{2}$, then for almost all segments $S$ of $\mathbb{R}^{2}$, the measure $\mu_{r}\left(S_{r}\right)$ does not converge to the length of $S$ when the resolution $r$ converges to 0 , where $S_{r}$ is the Bresenham discretization of the segment $S$ in $r \mathbb{Z}^{2}$. Moreover, the average errors of classical local definitions are estimated, and we define a new one which minimizes this error.
\end{abstract}

Keywords: Digital segments, local length estimation, frequency of factors, convergence.

\section{Introduction}

A digital curve is the discretization of a curve in $\mathbb{R}^{2}$. We investigate the local definitions of length of digital curves in $r \mathbb{Z}^{2}$ where $r$ is the resolution of discrete space.

The local definition of length is obtained by associating a weight $p(w)$ to each digital curve $w$ of size $m$, where the size of a digital curve is its cardinality minus one, ie its number of edges between consecutive points. If $\mathcal{C}(m)$ is the set of digital curves of size $m$, then any digital curve $\gamma_{r}$, in $r \mathbb{Z}^{2}$, can be obtained by concatenation of elements in $\mathcal{C}(m)$ with perhaps a digital curve $\varepsilon$ of size less than $m$. In other words $\gamma_{r}$ can be viewed as a word in $\mathcal{C}(m)^{*}$. $\varepsilon$. If $\gamma_{r}=w_{1} w_{2} \ldots w_{N} \varepsilon$ where $w_{i} \in \mathcal{C}(m)$ for all $i$, then we define the length of $\gamma_{r}$ by $\mu_{r, m, p}(\gamma)=r \sum_{i} p\left(w_{i}\right)$ (we neglect the contribution of digital curve $\varepsilon$ ).

Actually, we investigate the following problem:

Does there exist $m, p(\cdot)$ such that for any curve $\gamma$ of $\mathbb{R}^{2}$ the lengths $\mu_{r, m, p}\left(\gamma_{r}\right)$ converge to the length of $\gamma$ where $r$ tends to 0 ? (i.e. $\gamma_{r}$ is a discretization of $\gamma$ ).

In this paper, we study this problem for a particular class of curves: the set of segments in $\mathbb{R}^{2}$, moreover we suppose that the discretization operator $\delta_{r}$ restricted to the segments is the "Bresenham" discretization.

We consider the segment $S=\{(x, \alpha x+\beta) \mid A \leq x \leq B\}$ of $\mathbb{R}^{2}$ such that the slope $\alpha \in[0,1]$, the other cases could be studied by symmetry. Its "Bresenham" discretization $S_{r}=\delta_{r}(S) \subset r \mathbb{Z}^{2}$ is the set 


$$
S_{r}=r\left\{(X, Y) \in \mathbb{Z}^{2} \mid \frac{A}{r} \leq X \leq \frac{B}{r} \text { and } \alpha X+\frac{\beta}{r}-1<Y \leq \alpha X+\frac{\beta}{r}\right\} .
$$

We fix $m$ as a positive integer. As it has been explained for curves the segment $S_{r}$ can be seen as the word:

$$
w_{1} w_{2} \ldots w_{N} \varepsilon
$$

where $m \cdot N+\operatorname{Card}(\varepsilon)=\operatorname{Card}\left(S_{r}\right), w_{i}$ is a word of size $m$ and $\varepsilon$ is a word of size less than $m$. We call $\mathcal{S}_{m}$ the set of all such factors $w_{i}$ when $S$ describes all the segments. Figure 1 illustrates this situation.
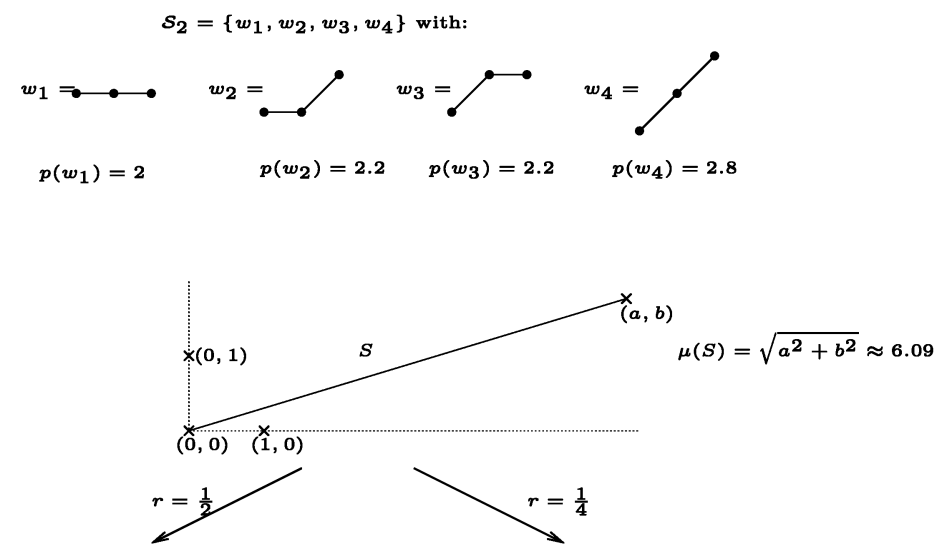

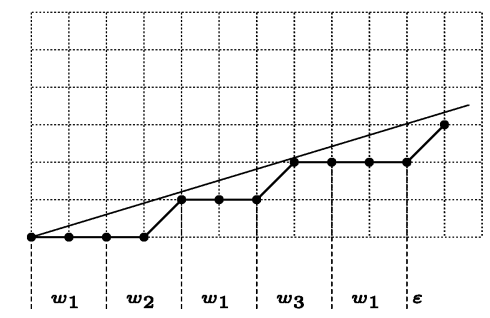

$\mu_{\frac{1}{2}, 2, p}(S)=\frac{1}{2}\left(3 p\left(w_{1}\right)+1 p\left(w_{2}\right)+1 p\left(w_{3}\right)\right)=5.2$

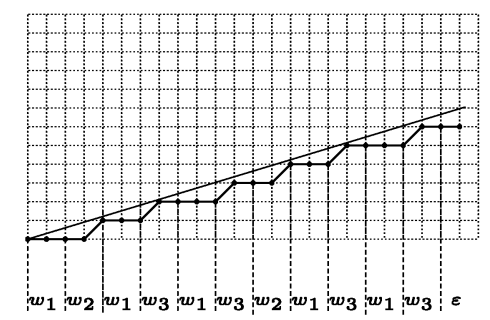

$\mu_{\frac{1}{4}, 2, p}(S)=\frac{1}{4}\left(6 p\left(w_{1}\right)+1 p\left(w_{2}\right)+4 p\left(w_{3}\right)\right)=5.75$

Fig. 1. A local estimator with $m=2$.

We construct $\mu_{r, m, p}$ as the local definition of measure by using a weight function $p: \mathcal{S}_{m} \longmapsto \mathbb{R}$. Then $\mu_{r, m, p}$ is defined by:

$$
\begin{aligned}
\mu_{r, m, p}\left(S_{r}\right) & =r\left(p\left(w_{1}\right)+p\left(w_{2}\right)+\ldots+p\left(w_{N}\right)\right) \\
& =r \sum_{w \in \mathcal{S}_{m}} n\left(w, S_{r}, r\right) p(w)
\end{aligned}
$$

where $n\left(w, S_{r}, r\right)$ is the number of $i$ between 1 and $N$ such that $w_{i}=w$. 
The central question of this paper can be formulated as the following: does there exist $m, p(\cdot)$ such that, for any segment $S$, the estimation $\mu_{r, m, p}\left(S_{r}\right)$ converges to the length of $S$ when the resolution $r$ converge to 0 ?

In this paper, we will prove that for almost all segments $S$, the estimation $\mu_{r, m, p}\left(S_{r}\right)$ does not converge to the length of $S$ when the resolution $r$ tends to 0 .

\section{Segments in $\mathbb{Z}^{2}$}

\subsection{Preliminaries}

Let $a \in \mathbb{R},\lfloor a\rfloor$ (resp. $\langle a\rangle$ ) denotes the integral part (resp. the fractional part) of $a$. So, $a=\lfloor a\rfloor+\langle a\rangle$ with $\lfloor a\rfloor \in \mathbb{Z},\lfloor a\rfloor \leq a<\lfloor a\rfloor+1$ and $0 \leq\langle a\rangle<1$. We also define $\lceil a\rceil=-\lfloor-a\rfloor$.

For example, $\left\lfloor\frac{7}{3}\right\rfloor=2,\left\lceil\frac{7}{3}\right\rceil=3$ and $\left\langle\frac{7}{3}\right\rangle=\frac{1}{3}$.

We have:

Property 1. Let $x, u$ be real numbers then:

$$
\lfloor x+u\rfloor-\lfloor x\rfloor=\left\{\begin{array}{l}
\lfloor u\rfloor \text { if }\langle x\rangle<1-\langle u\rangle \\
\lfloor u\rfloor+1 \text { otherwise. }
\end{array}\right.
$$

So, for all $\alpha, \beta \in \mathbb{R}$

$$
\lfloor\alpha(x+u)+\beta\rfloor-\lfloor\alpha x+\beta\rfloor=\left\{\begin{array}{l}
\lfloor\alpha u\rfloor \text { if }\langle\alpha x+\beta\rangle<1-\langle\alpha u\rangle \\
\lfloor\alpha u\rfloor+1 \text { otherwise. }
\end{array}\right.
$$

In this paper, we consider the discretization operator $\delta_{r}$ in $r \mathbb{Z}^{2}$ of the families of Bresenham's discretization.

Definition 1. Let $r>0$ and let $\alpha \in[0,1], \beta, A, B \in \mathbb{R}$.

Consider the segment $S=\{(x, \alpha x+\beta) \mid A \leq x \leq B\}$ of slope $\alpha$ and displacement $\beta$. $S_{r}=\delta_{r}(S)=\left\{r\left(X,\left\lfloor\alpha X+\frac{1}{r} \beta\right\rfloor\right) \mid\left\lceil\frac{A}{r}\right\rceil \leq X \leq\left\lfloor\frac{B}{r}\right\rfloor\right.$ and $\left.X \in \mathbb{Z}\right\}$ is the discretization of $S$ in $r \mathbb{Z}^{2}$.

The notion of digital segment is a central notion in this paper for the local definitions of length. This notion can be defined as particular subset of digital straight line (as in the Euclidean case) or by using the chaincodes.

Definition 2. Let $r>0$. Let $\alpha \in[0,1], \beta \in \mathbb{R}$ and $m \in \mathbb{N}^{*}$.

- Let $n \in \mathbb{Z}$. A subset $S=\left\{r\left(X,\left\lfloor\alpha X+\frac{1}{r} \beta\right\rfloor\right) \mid n \leq X \leq n+m\right.$ and $\left.X \in \mathbb{Z}\right\}$ is called a segment of size $m$ of $r \mathbb{Z}^{2}$. The point $r\left(n,\left\lfloor\alpha n+\frac{1}{r} \beta\right\rfloor\right)$ is called the starting point of $S$.

- A subset $S$ of $r \mathbb{Z}^{2}$ is a digital segment of size $m$ in $r \mathbb{Z}^{2}$, if there exists a segment $S^{\prime}$ of size $m$ of $r \mathbb{Z}^{2}$ such that $S=\left\{p-p_{0} \mid p \in S^{\prime}\right\}$, where $p_{0}$ is the starting point of $S$. So, a digital segment is a segment up to a translation.

- $\mathcal{S}_{r, m}$ is the set of all digital segments of size $m$ of $r \mathbb{Z}^{2}$ with the slope in $[0,1]$. 
If the slope $\alpha \in[0,1]$, then the notion of digital segment can be described by using the relative or the absolute chaincode as the following:

Definition 3. Let $\alpha \in[0,1]$ and $\beta \in \mathbb{R}$.

- The relative chaincode $v_{x, r, m}^{\alpha, \beta}$ of length $m$ at abscissa $x \in r \mathbb{Z}(x=r X$ where $X \in \mathbb{Z})$ is the word on $\{0,1\}$ defined by:

$$
v_{x, r, m}^{\alpha, \beta}(k)=\left\lfloor\alpha(X+k)+\frac{\beta}{r}\right\rfloor-\left\lfloor\alpha(X+k-1)+\frac{\beta}{r}\right\rfloor .
$$

for $0<k \leq m$.

- The absolute chaincode $w_{x, r, m}^{\alpha, \beta}$ is defined by:

$$
w_{x, r, m}^{\alpha, \beta}(k)=\left\lfloor\alpha(X+k)+\frac{\beta}{r}\right\rfloor-\left\lfloor\alpha X+\frac{\beta}{r}\right\rfloor
$$

for $0 \leq k \leq m$.

These two chaincodes are equivalent, since $v_{x, r, m}^{\alpha, \beta}(k)=w_{x, r, m}^{\alpha, \beta}(k)-w_{x, r, m}^{\alpha, \beta}(k-1)$ and $w_{x, r, m}^{\alpha, \beta}(k)=\sum_{l=1}^{k} v_{x, r, m}^{\alpha, \beta}(l)$.

We consider the set of absolute chaincodes for a given slope $\alpha$ and displacement $\beta$ :

$$
C_{r, m}^{\alpha, \beta}=\left\{w_{x, r, m}^{\alpha, \beta} \mid x \in r \mathbb{Z}\right\}
$$

and the set of all the absolute chaincodes

$$
A_{r, m}=\left\{w_{x, r, m}^{\alpha, \beta} \mid \alpha \in[0,1], \beta \in \mathbb{R}, x \in r \mathbb{Z}\right\} .
$$

So, $A_{r, m}=\bigcup_{\alpha \in[0,1], \beta \in \mathbb{R}} C_{r, m}^{\alpha, \beta}$ and $\operatorname{Card}\left(A_{r, m}\right)=\operatorname{Card}\left(\mathcal{S}_{r, m}\right)$.

In the following, we will prove that the set $C_{r, m}^{\alpha, \beta}$ is not depending of $\beta$ nor $r$ and the set $A_{r, m}$ and $\mathcal{S}_{r, m}$ are not depending of $r$.

\subsection{Some Combinatorial Properties of Digital Segments}

In this subsection, we consider a segment with the slope $\alpha \in[0,1]$ and the displacement $\beta \in \mathbb{R}$.

Definition 4. Let $m \in \mathbb{N}^{*} \cdot \mathcal{F}_{m}$ is the set of Farey numbers of order $m: \mathcal{F}_{m}=$ $\left\{\frac{p}{q} \mid 0 \leq p \leq q \leq m\right.$ and $\left.\neq 0\right\}$. The elements of $\mathcal{F}_{m}$ are called $m$-Farey numbers.

We recall properties about the structure of the chaincodes of a given line (see $[1,2])$. The first one is a direct consequence of Property 1:

Property 2. Let $x \in r \mathbb{Z}$. If $x=r X$ with $X \in \mathbb{Z}$ then

$$
w_{x, r, m}^{\alpha, \beta}(k)=\left\{\begin{array}{l}
\lfloor\alpha k\rfloor \text { if }\left\langle\alpha X+\frac{\beta}{r}\right\rangle<1-\langle\alpha k\rangle \\
\lfloor\alpha k\rfloor+1 \text { otherwise. }
\end{array}\right.
$$


We define $\left(B_{i}^{\alpha}\right)_{0 \leq i \leq m}$ as the sequence $(1-\langle\alpha k\rangle)_{0 \leq k \leq m}$ reordered increasingly, notice that $B_{m}^{\alpha}=1-a \cdot 0=1$. By convention we suppose $B_{-1}^{\alpha}=0$.

Property 3. $[1,2]$

- If $\alpha \in\left([0,1] \backslash \mathcal{F}_{m}\right)$, then $1-\langle\alpha i\rangle \neq 1-\langle\alpha j\rangle$ for all $i, j$ such that $-1 \leq i<$ $j \leq m$.

- The chaincode $w_{x, r, m}^{\alpha, \beta}$ at $x$ depends only on the position of the number $\left\langle\alpha X+\frac{\beta}{r}\right\rangle$ relatively to the elements of the sequence $\left(B_{i}^{\alpha}\right)_{-1 \leq i \leq m}$. So if $x=$ $r X, x^{\prime}=r X^{\prime} \in r \mathbb{Z}$ then $w_{x, r, m}^{\alpha, \beta}=w_{x^{\prime}, r, m}^{\alpha, \beta} \Longleftrightarrow \exists i \in\{-1,0, \ldots, m-1\}$ such that $\left\langle\alpha X+\frac{\beta}{r}\right\rangle,\left\langle\alpha X^{\prime}+\frac{\beta}{r}\right\rangle \in\left[B_{i}^{\alpha}, B_{i+1}^{\alpha}[\right.$.

- If $B_{i}^{\alpha}<B_{i+1}^{\alpha}$ then for all $\beta \in \mathbb{R}$ and $r>0$ there exists $X \in \mathbb{Z}$ such that $\left\langle\alpha X+\frac{\beta}{r}\right\rangle \in\left[B_{i}^{\alpha}, B_{i+1}^{\alpha}\left[\right.\right.$. So, the sets $C_{r, m}^{\alpha, \beta}$ do not depend on $\beta$ nor $r$ and will be denoted $C_{m}^{\alpha}$ and thus the set $A_{r, m}$ (resp. $\mathcal{S}_{r, m}$ ) does not depends on $r$ and will be denoted $A_{m}$ (resp. $\left.\mathcal{S}_{m}\right)$.

Moreover $\operatorname{Card}\left(C_{m}^{\alpha}\right)=\operatorname{Card}\left(\left\{i \mid 0<i<m\right.\right.$ and $\left.\left.B_{i}^{\alpha}<B_{i+1}^{\alpha}\right\}\right) \leq m+1$. Thus, if $\alpha \in\left([0,1] \backslash \mathcal{F}_{m}\right)$, then $\operatorname{Card}\left(C_{m}^{\alpha}\right)=m+1$.

Property 4. [1]

- $\mathcal{S}_{m}$ is the set of segments of $\mathbb{Z}^{2}$ with $(0,0)$ as starting point.

- $\operatorname{Card}\left(\mathcal{S}_{m}\right)=1+\sum_{i=1}^{m} \varphi(i)$ where $\varphi$ is the Euler's totient function $(\varphi(i)=$ $\operatorname{Card}(\{j \mid 1 \leq j<i$ and $i$ and $j$ are coprime $\}))$.

- $\operatorname{Card}\left(\mathcal{S}_{m}\right)=\frac{m^{3}}{\pi^{2}}+O\left(m^{2} \log (m)\right)$.

Definition 5. Let $\alpha \in\left([0,1] \backslash \mathcal{F}_{m}\right) . \sigma_{\alpha}$ is the permutation on $\{1, \ldots, m\}$ such that: $1-\left\langle\alpha \sigma_{\alpha}(i)\right\rangle<1-\left\langle\alpha \sigma_{\alpha}(i+1)\right\rangle$ for $1 \leq i<m$. So, $B_{i}^{\alpha}=1-\left\langle\alpha \sigma_{\alpha}(i)\right\rangle$ for all $1 \leq i \leq m$.

Lemma 1. Let $f, f^{\prime}$ be two consecutive m-Farey numbers and $\left.\alpha, \alpha^{\prime} \in\right] f, f^{\prime}[$. Then $\sigma_{\alpha}=\sigma_{\alpha^{\prime}}$. In other words, the function $\alpha \mapsto \sigma_{\alpha}$ is a constant function on ]$f, f^{\prime}\left[\right.$. Moreover, the function $\alpha \mapsto B_{i}^{\alpha}$ is an affine function on $] f, f^{\prime}[$.

The proof of Lemma 1 is omitted due to space constraints and is available in [3].

Theorem 1. Let $m \in \mathbb{N}^{*}$ and $0 \leq j<m$. Let $I \subseteq[0,1]$ be an interval. Then

$$
\lim _{r \rightarrow 0} \frac{\operatorname{Card}\left(\left\{X \in(m \mathbb{Z}+j) \mid\left\langle\alpha X+\beta_{r}\right\rangle \in I \text { and }\left\lceil\frac{A}{r}\right\rceil \leq X \leq\left\lfloor\frac{B}{r}\right\rfloor\right\}\right)}{\left\lfloor\frac{B}{r}\right\rfloor-\left\lceil\frac{A}{r}\right\rceil+1}=\frac{1}{m} \mu(I)
$$

where $\mu(I)$ is the length of the interval $I$.

The proof of Theorem 1 is analogous to the proof of Theorem 1.19 of [4] (Weyl's Theorem), and is given in [3]. 


\subsection{Local Definitions of Length of Digital Segments}

Let $m \in \mathbb{N}^{*}$. We construct the local definition of length by using a weight function $p: \mathcal{S}_{m} \longmapsto \mathbb{R}$ as the following:

Let $S=\{(x, \alpha x+\beta) \mid A \leq x \leq B\}$ be a segment in $\mathbb{R}^{2}$ with $\alpha \in[0,1]$, $\beta, A, B \in \mathbb{R}$, and let $r>0$.

Then $S_{r}=\delta_{r}(S)=r\left\{\left(X,\left\lfloor\alpha X+\frac{1}{r} \beta\right\rfloor\right) \mid\left\lceil\frac{A}{r}\right\rceil \leq X \leq\left\lfloor\frac{B}{r}\right\rfloor\right.$ and $\left.X \in \mathbb{Z}\right\}$.

Let $N(r)=\left\lfloor\frac{\left\lfloor\frac{B}{r}\right\rfloor-\left\lceil\frac{A}{r}\right\rceil+1}{m}\right\rfloor$.

So, $S_{r}$ can be seen as the word $w_{1, r} w_{2, r} \ldots w_{N(r), r} \varepsilon_{r}$ where $w_{i, r} \in \mathcal{S}_{m}$ for $i=$ $1, \ldots, N(r)$ and $\varepsilon_{r}$ a word of size less than $m$.

Consider $\mu_{r, m, p}\left(S_{r}\right)=r\left(p\left(w_{1, r}\right)+p\left(w_{2, r}\right)+\ldots+p\left(w_{N(r), r}\right)\right)$ as an approximation of the length of the segment $S$ (we neglect the contribution of $\varepsilon_{r}$ ).

Put, $D A_{m, p}(S)=\lim _{r \rightarrow 0}\left(\mu_{r, m, p}\left(S_{r}\right)\right)$

Definition 6. Let $j$ such that $0 \leq j<m$. The frequency $F_{j, r}^{\alpha, \beta, A, B}(w)$ of a word $w$ of length $m$ in the segment $\delta_{r}(S)=\left\{r\left(X,\left\lfloor\alpha X+\frac{1}{r} \beta\right\rfloor\right) \mid\left\lceil\frac{A}{r}\right\rceil \leq X \leq\right.$ $\left\lfloor\frac{B}{r}\right\rfloor$ and $\left.X \in \mathbb{Z}\right\}$ of $r \mathbb{Z}^{2}$ is defined by:

$$
F_{j, r}^{\alpha, \beta, A, B}(w)=\frac{\operatorname{Card}\left(\left\{X \in(m \mathbb{Z}+j) \mid\left\lceil\frac{A}{r}\right\rceil \leq X \leq\left\lfloor\frac{B}{r}\right\rfloor \text { and } w_{x, r, m}^{\alpha, \beta}=w\right\}\right)}{\left\lfloor\frac{B}{r}\right\rfloor-\left\lceil\frac{A}{r}\right\rceil+1} .
$$

Lemma 2. Let $\alpha \in[0,1]$ be an irrational number, $\beta, A, B \in \mathbb{R}, w \in C_{m}^{\alpha}, 0 \leq$ $j<m$ and $i$ as in Property 3. Then

$$
F_{j}^{\alpha, \beta, A, B}(w)=\lim _{r \rightarrow 0} F_{j, r}^{\alpha, \beta, A, B}(w)=\frac{1}{m}\left(B_{i}^{\alpha}-B_{i-1}^{\alpha}\right) .
$$

In particular $F_{j}^{\alpha, \beta, A, B}(w)$ does not depend on $j, \beta, A$ and $B$, and will be denoted $F^{\alpha}(w)$ in the following.

Proof. By Property 3 we have:

$$
\begin{aligned}
& F_{j}^{\alpha, \beta, A, B}(w)=\lim _{r \rightarrow 0} \frac{\operatorname{Card}\left(\left\{X \in(m \mathbb{Z}+j) \mid\left\lceil\frac{A}{r}\right\rceil \leq X \leq\left\lfloor\frac{B}{r}\right\rfloor \text { and } w_{x, r, m}^{\alpha, \beta}=w\right\}\right)}{\left\lfloor\frac{B}{r}\right\rfloor-\left\lceil\frac{A}{r}\right\rceil+1} \\
& =\lim _{r \rightarrow 0} \frac{\operatorname{Card}\left(\left\{X \in(m \mathbb{Z}+j) \mid\left\lceil\frac{A}{r}\right\rceil \leq X \leq\left\lfloor\frac{B}{r}\right\rfloor \text { and }\left\langle\alpha X+\frac{1}{r} \beta\right\rangle \in\left[B_{i-1}^{\alpha}, B_{i}^{\alpha}[\}\right)\right.\right.}{\left\lfloor\frac{B}{r}\right\rfloor-\left\lceil\frac{A}{r}\right\rceil+1}
\end{aligned}
$$

So, by Theorem $1, F_{j}^{\alpha, \beta, A, B}(w)=\frac{1}{m}\left(B_{i}^{\alpha}-B_{i-1}^{\alpha}\right)$.

Remark 1. This lemma is wrong for rational slopes. For example, if we consider the line $y=\left\lfloor\frac{1}{2} x\right\rfloor$, then the frequency of the word $w=(0,0,1)$ is 1 . But this word corresponds to the interval $\left[B_{-1}^{\frac{1}{2}}, B_{0}^{\frac{1}{2}}\right.$ [ whose length is $\frac{1}{2}$.

Theorem 2. Let $f, f^{\prime}$ be two consecutive $m$-Farey numbers. There exist $u, v$ such that $D A_{m, p}(S)=(B-A)(u \alpha+v)$ for all segments $S=\{(x, \alpha x+\beta) \mid A \leq$ $x \leq B\}$, such that $\left.\alpha \in(] f, f^{\prime} \backslash \backslash \mathbb{Q}\right)$ (i.e. $\alpha$ is an irrational numbers between the two $m$-Farey numbers $\left.f, f^{\prime}\right)$.

In other words, $D A_{m, p}($.$) is piecewise affine function in \alpha$ for $\alpha \in([0,1] \backslash \mathbb{Q})$. 
Proof. We suppose the weight function $p: \mathcal{S}_{m} \mapsto \mathbb{R}$ which associates to each chaincode $w$ of size $m$ a weight $p(w)$. The digital segment $S_{r}$ can be seen as the word:

$$
w_{1, r} w_{2, r} \ldots w_{N(r), r} \varepsilon_{r}
$$

where $N(r)=\left\lfloor\frac{\left\lfloor\frac{B}{r}\right\rfloor-\left\lceil\frac{A}{r}\right\rceil+1}{m}\right\rfloor, w_{i, r} \in \mathcal{S}_{m}$ for $i=1, \ldots, N(r)$ and $\varepsilon_{r}$ is a word of length less than $m$.

So, the approximated length of the digital segment $S_{r}$ is:

$$
\begin{aligned}
\mu_{r, m, p}\left(S_{r}\right) & =r\left(p\left(w_{1, r}\right)+p\left(w_{2, r}\right)+\ldots+p\left(w_{N(r), r}\right)\right. \\
& =r \sum_{w \in \mathcal{S}_{m}} n\left(w, S_{r}, r\right) p(w)
\end{aligned}
$$

where $n\left(w, S_{r}, r\right)=\operatorname{Card}\left(\left\{X \in m \mathbb{Z} \mid\left\lceil\frac{A}{r}\right\rceil \leq X \leq\left\lfloor\frac{B}{r}\right\rfloor\right.\right.$ and $\left.\left.w_{x, r, m}^{\alpha, \beta}=w\right\}\right)$ which is the number of $i$ such that $w_{i}^{r}=w$.

So,

$$
\begin{aligned}
D A_{m, p}(S) & =\lim _{r \rightarrow 0} r \sum_{w \in \mathcal{S}_{m}} n\left(w, S_{r}, r\right) p(w) \\
& =\lim _{r \rightarrow 0} r\left(\left\lfloor\frac{B}{r}\right\rfloor-\left\lceil\frac{A}{r}\right\rceil+1\right) \sum_{w \in \mathcal{S}_{m}} \frac{n\left(w, S_{r}, r\right)}{\left\lfloor\frac{B}{r}\right\rfloor-\left\lceil\frac{A}{r}\right\rceil+1} p(w) \\
& =(B-A) \sum_{w \in \mathcal{S}_{m}} F^{\alpha}(w) p(w) .
\end{aligned}
$$

So, according to Lemma 1 and Lemma $2, D A_{m, p}(S)$ is an affine function on $\alpha$ if $\alpha$ is irrational numbers between two consecutive $m$-Farey numbers.

Corollary 1. There are at most $\left(2 \sum_{i=1}^{m} \varphi(i)\right)$ irrational numbers $\alpha \in[0,1]$ such that $D A_{m, p}(S)=$ length $(S)$ where $S=\{(x, \alpha x+\beta) \mid A \leq x \leq B\}$ (i.e. length $(S)$ is the length of the segment $S$ and $\varphi$ is the Euler's totient function).

Proof. We consider an interval ] $f, f^{\prime}$ [ bordered by two consecutive $m$-Farey numbers. By the previous theorem, there exist $u, v \in \mathbb{R}$ such that the estimated length of the segment $S=\{(x, \alpha x+\beta) \mid A \leq x \leq B\}$ is $D S_{m, p}(S)=(B-$ $A)(u \alpha+v)$ for the irrational slopes $\alpha$. The exact length of $S$ is length $(S)=$ $(B-A) \sqrt{1+\alpha^{2}}$. But the equation

$$
\begin{aligned}
D S_{m, p}(S)=\text { length }(S) & \Leftrightarrow\left(1+\alpha^{2}\right)=(u \alpha+v)^{2} \\
& \Leftrightarrow\left(u^{2}-1\right) \alpha^{2}+2 u v \alpha+\left(v^{2}-1\right)=0
\end{aligned}
$$

has more than two solutions only when $u^{2}-1=0, u v=0, v^{2}-1=0$ which never happens. So the estimated length can be equal to the exact length for only two values on each interval. There are exactly $\sum_{i=1}^{m} \varphi(i)$ such intervals. So the estimated length is exact for at most $2 \sum_{i=1}^{m} \varphi(i)$ slopes. 
Remark 2. Theorem 2 and Corollary 1 imply that: for any $m \in \mathbb{N}^{*}$ and any weight $p(\cdot)$ the set of slopes $\alpha \in[0,1]$, such that the length of segments $S$ of slope $\alpha$ is equal to $D S_{m, p}(S)$ is at most a countable set. So, for any $m \in \mathbb{N}^{*}$, for almost all segments $S$ of slope $\alpha \in[0,1]$, length $(S) \neq D A_{m, p}(S)$.

\section{$3 \quad$ Examples of Estimators for Segments}

In this section we compare different local estimators. Table 1 gives for each method, the irrational slopes for which the asymptotic length is exact (By Corollary 1 for every local estimator there is always a finite number of such slopes), and the root mean square error. This last is given by the formula:

$$
R M S E=\sqrt{\frac{\int_{0}^{1}\left(l_{\text {est }}(\alpha)-l_{\text {real }}(\alpha)\right)^{2} D(\alpha) \mathrm{d} \alpha}{\int_{0}^{1} D(\alpha) \mathrm{d} \alpha}}
$$

where $l_{\text {est }}(\alpha)=D A_{m, p}(S(\alpha))$ is the estimated length of the segment $S(\alpha)=$ $\{(x, \alpha x) \mid 0 \leq x \leq 1\}, l_{\text {real }}(\alpha)$ is the real length: $l_{\text {real }}(\alpha)=\sqrt{1+\alpha^{2}}, D(\alpha)$ is the density of the lines of slope $\alpha$. In the following we suppose that the distribution of the angles of the lines is uniform, which means: $D(\alpha)=\frac{\mathrm{d} \arctan \alpha}{\mathrm{d} \alpha}=\left(1+\alpha^{2}\right)^{-1}$. In the previous section we have computed $l_{\text {est }}(\alpha)$ for every irrational $\alpha$, so we can compute precisely the RMSE for every local estimator of length.

\subsection{Some Classical Estimators}

We have considered three classical kinds of estimators: Freeman's estimator ([5]), Chamfer estimators ([6]) and BLUE estimators ([7]). The weights of these estimators and their RMSE are given in Table 1. For more details see [3].

\subsection{Minimum RMSE Estimator}

In this paragraph we propose estimators which minimize the RMSE. In fact Formula (1) and Lemma 2 permit easily to express the RMSE in the weights:

$$
\begin{aligned}
\left(\int_{0}^{1} D(\alpha) \mathrm{d} \alpha\right) R M S E^{2}=\int_{0}^{1}\left(l_{\text {est }}(\alpha)-l_{\text {real }}(\alpha)\right)^{2} D(\alpha) \mathrm{d} \alpha \\
=\int_{0}^{1}\left(\left(\sum_{w \in \mathcal{S}_{m}} F^{\alpha}(w) p(w)\right)-\sqrt{1+\alpha^{2}}\right)^{2} D(\alpha) \mathrm{d} \alpha \\
=\sum_{\left(w_{1}, w_{2}\right) \in\left(\mathcal{S}_{m}\right)^{2}}\left(p\left(w_{1}\right) p\left(w_{2}\right) \int_{0}^{1} F^{\alpha}\left(w_{1}\right) F^{\alpha}\left(w_{2}\right)\right) D(\alpha) \mathrm{d} \alpha \\
\quad-2 \sum_{w \in \mathcal{S}_{m}}\left(p(w) \int_{0}^{1} F^{\alpha}(w) \sqrt{1+\alpha^{2}} D(\alpha) \mathrm{d} \alpha\right)+\int_{0}^{1}\left(1+\alpha^{2}\right) D(\alpha) \mathrm{d} \alpha
\end{aligned}
$$


so the function $(p(w))_{w \in \mathcal{S}_{m}} \mapsto R M S E^{2}$ is a quadratic form. It is positive so it reaches its minimum for some values of weights which give the minimum RMSE estimators.

Each last line of the two parts of Table 1 gives these weights for the wordlengths $m=1,2$ and the corresponding errors. (computed with the formal calculus system Maple)

Figure 2 gives the estimated length when the resolution tends to zero for three different estimators. By definition, the minimum RMSE estimator is the more closed to the real length.

Table 1. Comparison of length estimators in the plane.

\begin{tabular}{|c|c|c|c|c|}
\hline & $\mathrm{p}(00)$ & $\mathrm{p}(01)$ & $\begin{array}{c}\text { asymptotic root } \\
\text { mean square error }\end{array}$ & slopes with no errors \\
\hline Freeman & 1 & $\sqrt{2}$ & 0.066143 & $\{0,1\}$ \\
\hline Chamfer 3-4 & 1 & $\frac{4}{3}$ & 0.042255 & $\left\{0, \frac{3}{4}\right\}$ \\
\hline$\overline{\mathrm{BLUE}}$ & 1.059416 & 1.183276 & 0.084863 & $\{0.510130\}$ \\
\hline $\begin{array}{l}\text { minimum } \\
\text { RMSE }\end{array}$ & 0.941246 & 1.351320 & 0.026524 & $\{0.184382,0.743633\}$ \\
\hline
\end{tabular}

$m=2$

\begin{tabular}{|c|c|c|c|c|c|}
\hline & $\mathrm{p}(000)$ & $\mathrm{p}(001), \mathrm{p}(011)$ & $\mathrm{p}(012)$ & $\begin{array}{c}\text { asymptotic root } \\
\text { mean square error }\end{array}$ & $\begin{array}{c}\text { irrational slopes } \\
\text { with no errors }\end{array}$ \\
\hline Chamfer 5-7-11 & 2 & $\frac{22}{10}$ & $\frac{28}{10}$ & 0.011875 & none \\
\hline BLUE & 2.037583 & 2.226499 & 2.583985 & 0.043534 & $\{0.480972\}$ \\
\hline $\begin{array}{c}\text { minimum } \\
\text { RMSE }\end{array}$ & 1.958843 & 2.205554 & 2.811569 & 0.007466 & $\begin{array}{c}0.106259,0.408328, \\
0.634893,0.897172\}\end{array}$ \\
\hline
\end{tabular}

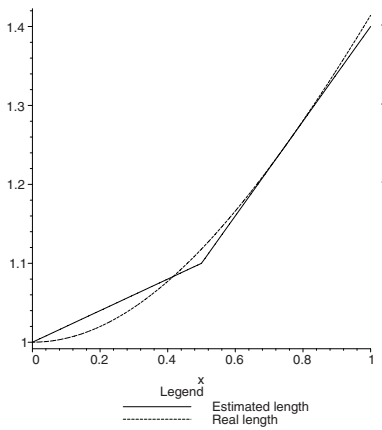

Chamfer 5-7-11

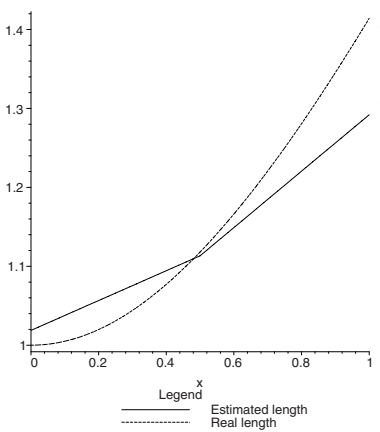

BLUE

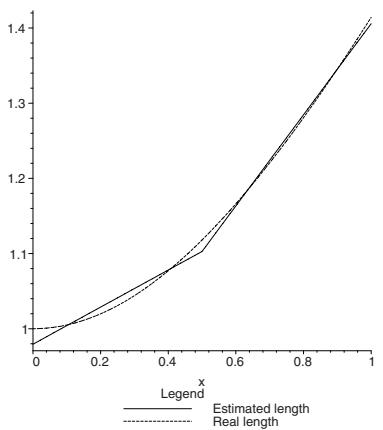

Minimum RMSE

Fig. 2. Length approximated by three estimators in function of the slope of the segment. $(m=2)$. 


\section{Conclusion}

In this paper we have proved that local definitions of digital length cannot be used to estimate the length of continuous curves because we does not have the convergence of such measurements to the searched length when the resolution tends to infinity, even if we restrict the curves to the segments. But, of course it does not mean that the discretizations of the curves do not permit to compute a good estimation of the length of the continuous curve. For example in [8] the authors measure the length of a curve by summing the length of segments included in the curve. They prove that the limit length when the resolution tends to infinity is the searched length if the curve satisfies some regularity properties. See also [9] for a comparison between different estimators.

\section{References}

1. Mignosi, F.: On the number of factors of Sturmian words. Theoret. Comput. Sci. 82 (1991) 71-84

2. Gérard, Y.: Contribution à la Géométrie Discrète. PhD thesis, Université Clermont 1 (1999)

3. Tajine, M., Daurat, A.: On local definitions of digital curves. Technical report, LSIIT (2003) extended version with proof.

4. Drmota, M., Tichy, R.F.: Sequences, discrepancies and applications. Lecture Notes in Mathematics 1651. Springer-Verlag (1997)

5. Freeman, H.: Boundary encoding and processing. In Lipkin, B.S., Rosenfeld, A., eds.: Picture Processing and Psychopitorics. (1970)

6. Borgefors, G.: Distance transformations in digital images. Computer Vision, Graphics, and Image Processing 34 (1986) 344-371

7. Dorst, L., Smeulders, A.W.M.: Best linear unbiased estimators for properties of digitized straight lines. IEEE Transactions on Pattern Analysis and Machine Intelligence 8 (1986) 276-282

8. Coeurjolly, D., Debled-Rennesson, I., Teytaud, O.: Segmentation and length estimation of 3D discrete curves. Lecture Notes in Computer Science 2243 (2001) 299-317 (DGCI' 2001)

9. Coeurjolly, D., Klette, R.: A comparative evaluation of length estimators. Technical Report, CITR-TR-105, University of Auckland (2001)

10. Berthé, V.: Fréquences des facteurs des suites sturmiennes. Theoret. Comput. Sci. 165 (1996) 295-309

11. Dorst, L., Smeulders, A.W.M.: Discrete straight line segments: Parameters, primitives and properties. In Melter, R., Bhattacharya, P., Rosenfeld, A., eds.: Vision Geometry, series Contemporary Mathematics. Volume 119., AMS (1991) 45-62

12. Dorst, L., Smeulders, A.N.M.: Discrete representation of straight lines. IEEE Transactions on Pattern Analysis and Machine Intelligence 6 (1984) 450-463

13. Berenstein, C.A., Kanal, L.N., Lavine, D., Olson, E.C.: A geometric approach to subpixel registration accuracy. Computer Vision, Graphics, and Image Processing 40 (1987) 334-360

14. Amanatides, J., Woo, A.: A fast voxel traversal algorithm for ray tracing. In Maréchal, G., ed.: Eurographics '87, Elsevier (1987) 3-10

15. Borgefors, G.: Distance transformations in arbitrary dimensions. Computer Vision, Graphics, and Image Processing 27 (1984) 321-345 